Z. Klin. Chem. Klin. Biochem.

11. Jg. 1973, S. 155-158

\title{
Die Lactatdehydrogenase-Isoenzymmuster des Myokards und Reizleitungssystems des Kalbsherzens
}

\author{
Von G. DöLken und K. Schumacher \\ Aus der Mediqinischen Universitätsklinik (Direktor: Prof. Dr. R. Gross) Köln
}

(Eingegangen am 12. September/13. November 1972)

Die $\mathrm{LDH}^{1}$ )-Isoenzymmuster des Myokards und Reizleitungssystems des Kalbsherzens wurden mit Hilfe der Polyacrylamid-Disc-Elektrophorese bestimmt. In beiden Organanteilen überwiegen die Isoenzyme LDH-1, LDH-2 und LDH-3. Es konnte der Einfluß der Lactatkonzentration in der Färbelösung auf das LDH-Isoenzymmuster gezeigt werden. Mit hohen Substratkonzentrationen lassen sich in beiden Organextrakten auch die kathodischen Isoenzyme LDH-4 und LDH-5 nachweisen. Dicser Befund unterstreicht die Bedeutung methodischer Faktoren bei der Bestimmung von LDH-Isoenzymmustern. Die Isoenzyme LDH-1, LDH-2 und LDH-3 des Myokards wurden chromatographisch getrennt und auf ihre Substrataffinität zu Pyruvat und Lactat untersucht. Mögliche Beziehungen zivischen dem LDH-Isoenzymmuster der Purkinjezellen und ihrem Stoffwechseltyp, insbesondere der Stoffwechsclaktivität, werden diskutiert.

\section{Lactate debydrogenase-isozyme patterns in the myocardium and Purkinje cells of the calf beart}

Lactate dehydrogenase-isozyme patterns in the myocardium and Purkinje cells of the calf heart were determined by disc-electrophoresis in polyacrylamide gel. In both organs the predominant isozymes are LDH 1, LDH 2 and LDH 3. It was demonstrated that the concentration of lactate in the staining solution has an marked infuence on the observed isozyme pattern. The cathodic isozymes LDH 4 and LDH 5 could be detected at high substrate concentrations in both organs. These results underline the importance of methodological conditions for the determination of lactate dehydrogenase-isozyme patterns. LDH 1, LDH 2 and LDH 3 of the myocardium were seperated by ion exchange chromatography and their kinetic properties were determined. Possible relationships between the metabolism of the Purkinje-cells (mainly their total metabolic activity) and their lactate dehydrogenase-isozyme pattern are discussed.

Die quantitative Bestimmung von LDH-Isoenzymen hat die Möglichkeiten klinischer Labordiagnostik durch die Erfassung pathologischer, teils organtypischer Veränderungen des LDH-Isoenzymmusters im Serum $(1-5)$ und im bioptisch gewonnenen Gewebsmaterial $(6,7)$ wesentlich erweitert. Die unterschiedlichen enzymkinetischen Eigenschaften der LDH-Isoenzyme und ihre unterschiedliche Verteilung in Organen mit verschiedenem Stoffwechseltyp ließen Beziehungen zwischen dem LDH-Isoenzymmuster einer Zelle und ihrem Stoffwechsel vermuten $(8,9,10)$. Befunde an Erythrocyten und Thrombocyten schienen einer direkten Beziehung dieser Art jedoch zu widersprechen (11).

Wir bestimmten deshalb die LDH-Isoenzymmuster im Reizleitungssystem und Myokard des Kalbsherzens, da beide Organanteile aus genetisch nah verwandten Zellen bestehen und einen unterschiedlichen Stoffwechsel besitzen sollen. Ferner untersuchten wir den Einfluß einiger methodischer Faktoren auf das LDHIsoenzymmuster.

\section{Methodik}

Die Bioclsemikalien wurden von der Fa. Boehtinger Mannheim bezogen.

Alle übrigen Chemikalien waren p. a. Substanzen der Firmen E. Merck, Darmstadt und Sigma Chem. Co. Ltd., London.

Als Untersiucbungsmaterial wurden Kalbs- und Rinderherzen verwendet, díe zwei Stunden post mortem präpariert wurden. Der linke Schenkel des Reizleitungssystems und Gewebe aus der linken Ventrikelwand wurden mit der Schere zerkleinert und bezogen auf die Gewebseinwaage 1:19 mit $50 \mathrm{mmol} / 1$ Triäthanolamin-HCl-Puffer $\mathrm{pH} 7,4 \mathrm{mit} 1 \mathrm{mmol} / \mathrm{l}$ EDTA verdünnt, $2 \mathrm{mal}$ $30 \mathrm{~s}$ im Eisbad homogenisiert (Sorvall, Omni-Mixer), nach $\mathrm{Zu}-$ satz von $2 \mathrm{ml}$ gesättigter Digitoninlösung $30 \mathrm{~min}$ nachextrahiert und $10 \mathrm{~min}$ bei $500 \mathrm{~g}$ zentrifugiert. Der Überstand war das Ausgangsmaterial für alle weiteren Studien.

Die elektrophoretische Trennung der LDH-Isoenzyme wurde nach dem von DiETz und Lubrano angegebenen Verfahren (12) der Disc-Elektrophorese in Polyacrylamid-Gel durchgeführt (13). Anschließend wurden die Gele in folgendem Färbemedium bei $37^{\circ} \mathrm{C}$ inkubiert: $50 \mathrm{mg}$ Natriumcyanid, $1,8 \mathrm{ml}$ NatriumlactatLösung $(700 \mathrm{~g} / \mathrm{l}), 2,5 \mathrm{mg}$ Phenazinmethosulfat, $25 \mathrm{mg}$ Tetrazoliumblauchlorid, $75 \mathrm{mg}$ NAD in $100 \mathrm{ml} 50 \mathrm{mmol} / \mathrm{l} \mathrm{Natrium}$ phosphatpuffer $\mathrm{pH} 8,5 \mathrm{mit} 1 \mathrm{mmol} / \mathrm{l}$ EDTA. Zur Bestimmung der Substratabhängigkeit des LDH-Isoenzymmusters wurden die Gele mit Färbelösungen unterschiedlicher Lactatkonzentration (3-870 mmol/l L-Lactat) inkubiert. Das $\mathrm{pH}$ des Färbemediums blieb konstant, die Leitfähigkeit änderte sich um das sechsfache.

Die LDH-Isoenzymbanden wurden mit einem Densitometer (Vitatron, Dieren, Niederlande) ausgemessen. Die Extinktion wurde bei $546 \mathrm{~nm}$ registriert, die Auswertung erfolgte mit Hilfe eines automatischen Integrators (14).

Die cbromatograpbische Trennung der LDH-Isoenzyme des Myokards erfolgte an DEAE-Cellulose (Scrva, Heidelberg). Die Höhe der Säulenpackung betrug $200 \mathrm{~mm}$, der Durchmesser $10 \mathrm{~mm} .20 \mathrm{~g}$ Herzmuskelgewebe wurden in $40 \mathrm{ml} 50 \mathrm{mmol} / 1$ TriäthanolaminHCl-Puffer pH 7,4 mit $1 \mathrm{mmol} / 1$ EDTA $2 \mathrm{mal} 30 \mathrm{~s}$ homogenisiert, nach Zusatz von $6 \mathrm{ml}$ gesättigter Digitoninlösung $30 \mathrm{~min}$ nachextrahiert und bei $500 \mathrm{~g}$ zentrifugiert. Mit dem Uberstand wurde zur Vorfraktionierung eine Ammoniumsulfatfällung durchgcführt. Das Sediment bei ciner Sättigung von $30-80 \%$ wurde nach Dialyse gegen den Elutionspuffer, $5 \mathrm{mmol}$ Tris- $\mathrm{HCl} \mathrm{pH} 8,3 \mathrm{mit}$ $1 \mathrm{mmol}$ EDTA, auf $5 \mathrm{ml}$ cingeengt, cin zweites $\mathrm{Mal}$ gegen den Elutionspuffer dialysiert und auf dic Säulc aufgetragen. Das

1) Abkürzung: $\mathrm{LDH}=$ Lactatdehydrogenase (EC 1.1.1.27). 
Eluat wurde in Fraktionen zu $3 \mathrm{ml}$ gesammelt. Die Elution mit dem Startpuffer erfolgte solange, bis LDH-4 und LDH-5 vollständig eluiert waren. Dann wurden dem Elutionspuffer steigende Natriumchloridkonzentrationen $(0,1-0,2 \mathrm{~mol} / \mathrm{l})$ zugesetzt.

Die Proteinkonzentration in den Fraktionen wurde spektrophotometrisch durch Messung der Absorption bei $280 \mathrm{~nm}$ bestimmt, die Ionenstärke durch Messung der Leitfähigkeit.

Die $L D H$-Aktivität wurde im optischen Test durch Messung der Extinktionsabnahme bei $340 \mathrm{~nm}$ bestimmt. Der Testansatz enthielt $5 \mu \mathrm{mol}$ Pyruvat und $0,4 \mu \mathrm{mol} \mathrm{NADH}$ in einem Gesamtvolumen von $2 \mathrm{ml} 50 \mathrm{mmol} / 1$ Triäthanolamin-HCl-Puffer $\mathrm{pH} 7,4$ mit $1 \mathrm{mmol} / \mathrm{l}$ EDTA.

Die chromatographisch isolierten LDH-IsoenzJ'me wurden mit der Polyacrylamid-Disc-Elektrophorese identifiziert. Ihre kinetischen Eigenschaften wurden im optischen Test mit Pyruvat $(0,0025-25 \mathrm{mmol} / \mathrm{l})$ und Lactat $(0,05-550 \mathrm{mmol} / \mathrm{l})$ bei $37^{\circ} \mathrm{C}$ bestimmt. Die eingesetzten Coenzymmengen waren $0,4 \mu \mathrm{mol}$ NADH bzw. $10 \mu \mathrm{mol}$ NAD im Bestimmungsansatz (s. o.).

\section{Ergebnisse}

Nach Auftrennung der Extrakte des Myokards und linken Schenkels des Reizleitungssystems von Kalbsund Rinderherzen mit der Polyacrylamid-Disc-Elektrophorese ließen sich bei spezifischer Anfärbung drei Banden enzymatischer Aktivität nachweisen (Abb. 1). Die Ergebnisse dieser Untersuchungen wurden zunächst für Kalbs- und Rinderherzen getrennt ausgewertet. Mit dem t-Test nach STUDENT fanden wir keine signifikanten Unterschiede zwischen beiden Kollektiven. Die relativen Anteile der einzelnen LDH-Isoenzyme an der LDH-Gesamtaktivität betragen im Myokard $(\mathrm{n}=22): \mathrm{LDH}-1=33,4 \pm 7,0, \mathrm{LDH}-2=52,7 \pm 4,9$, LDH-3 $=13,9 \pm 6,0$ rel\% und im Reizleitungssystem $(\mathrm{n}=18): \mathrm{LDH}-1=56,1 \pm 6,5, \mathrm{LDH}-2=40,1 \pm 4,3$ und $\mathrm{LDH}-3=3,8 \pm 1,8 \mathrm{rel} \%$. Der Vergleich der LDH-Isoenzymverteilung im Reizleitungssystem und Myokard ergab somit signifikante Differenzen im Gehalt aller Isoenzyme $(p<0,005)$.

Die in Abbildung 1 wiedergegebenen spezifischen Anfärbungen wurden bei einer Lactatkonzentration von $54 \mathrm{mmol} / \mathrm{l}$ durchgeführt. Den Einfluß unterschiedlicher Lactatkonzentrationen in der Färbelösung auf das LDH-Isoenzymmuster beider Organanteile zeigt Abbildung 2. Ubereinstimmend nehmen in den Zymogrammen beider Organextrakte die Aktivitätsanteile der anodischen Isoenzyme mit steigender Lactatkonzentration $a b$, während die kathodischen Isoenzymaktivitäten stärker angefärbt werden. Mit sehr hohen Lactatkonzentrationen konnten in beiden Organextrakten auch die Isoenzyme LDH-4 und LDH-5 nachgewiesen werden.

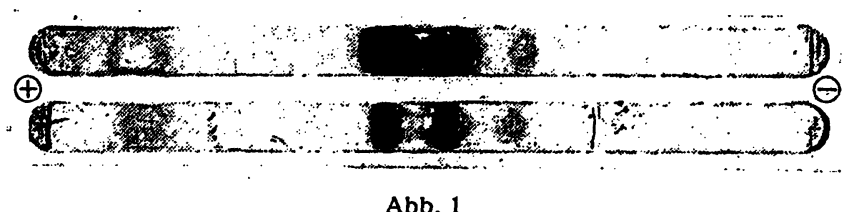

Die LDH-Isoenzymmuster des Reizleitungșsystems (oberes Gel) und des Myokards (unteres Gel) des Kalbsherzens nach Auftrennung der Organextrakte in der Polyacrylamid-Disc-Elektrophorese und Nach-
weis der Isoenzyme mit der Tetrazoliumfärbung (Auftragsstelle und weis der Isoenzyme mit der Tetrazoliumfär
Kathode rechts)
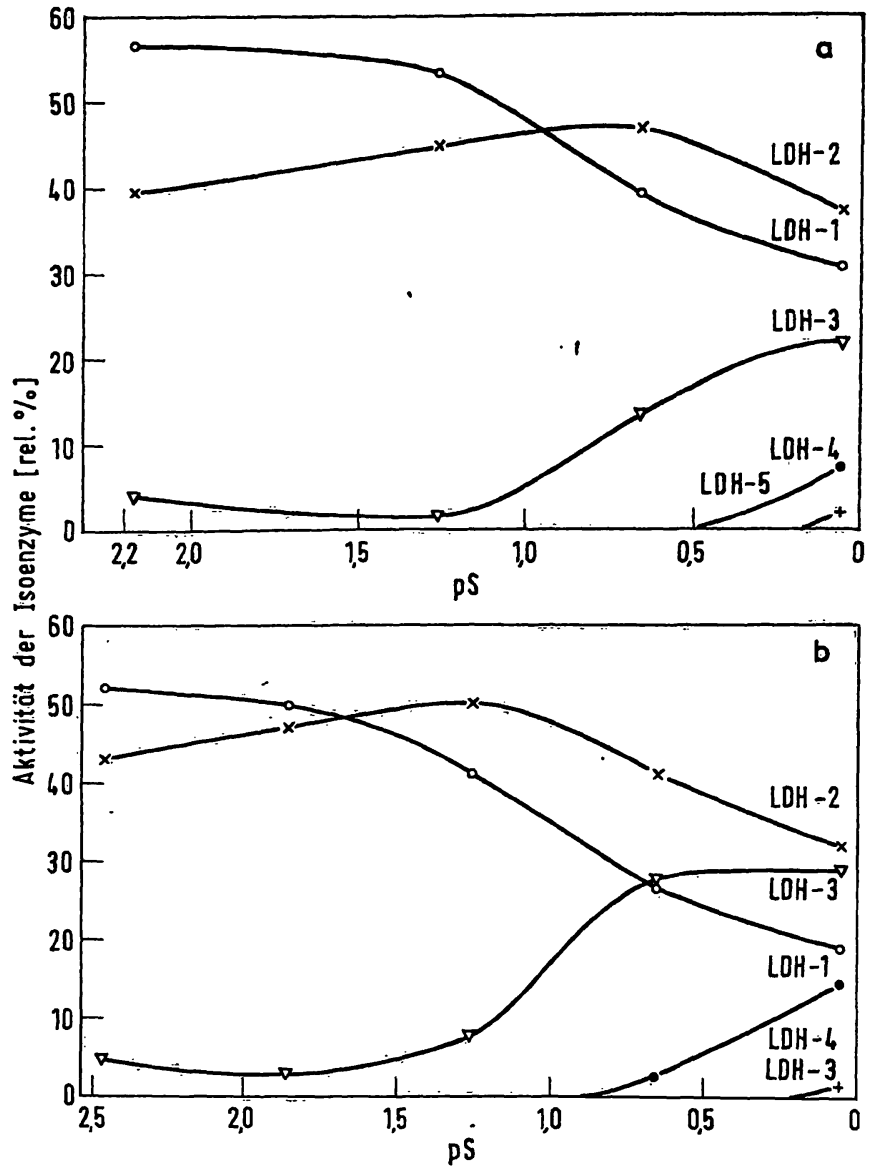

Abb. 2

Die Abhängigkeit des LDH-Isoenzymmusters a) des Reizleitungssystems und b) Arbeitsmyokards des Kalbsherzens von der Lactatkonzentration ( $\mathrm{pS}=-\log \mathrm{mol} / \mathrm{l}$ ) in der Färbelöșung

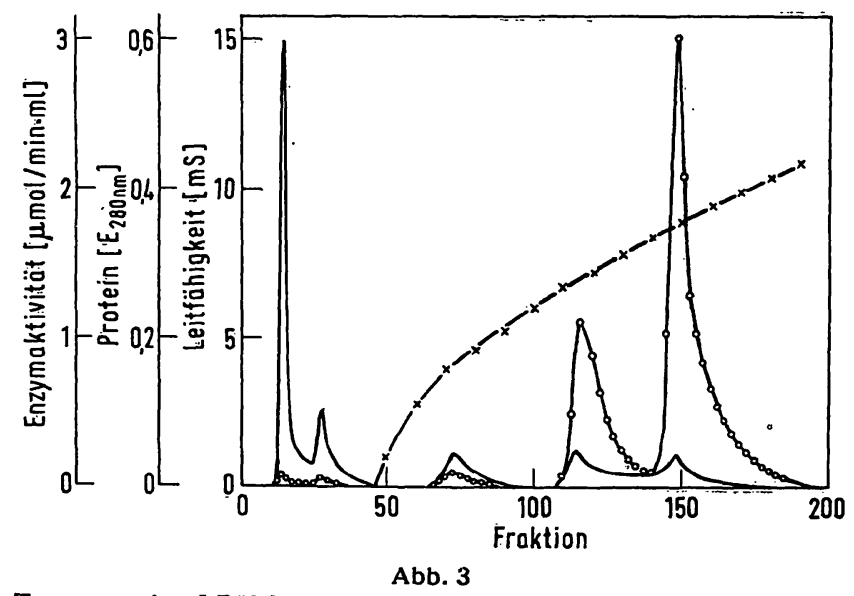

Trennung der LDH-Isoenzyme des Myokards des Kalbsherzens an

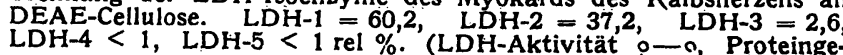
halt - , Leittähigkeit $x-x$ )

Bei der chromatographischen Trennung der LDHIsoenzyme des Myokards an DEAE-Cellulose wurden die Isoenzyme LDH-4 und LDH-5 vom Ionenaustauscher nicht adsorbiert und erschienen als fast einheitliche Bande im Eluat (Abb. 3). Nach vollständiger Elution der beiden kathodischen Isoenzyme konnten mit steigenden Natriumchloridkonzentrationen LDH-3, LDH-2 und LDH-1 eluiert werden. Chromatographisch fanden wir folgende Isoenzymverteilung: $\mathrm{LDH}-1=$ $60,2, \mathrm{LDH}-2=37,2, \mathrm{LDH}-3=2,6, \mathrm{LDH}-4$ und LDH-5 $<1$ rel\%. Die Identifizierung der Isoenzyme LDH-1, 
LDH-2 und LDH-3 mit der Polyacrylamid-DiscElektrophorese ergab jeweils nur eine Bande (13).

Kinetische Untersuchungen wurden nur mit den chromatographisch getrennten Isoenzymen LDH-1, LDH-2 und LDH-3 durchgeführt, da sie im Myokard den Hauptanteil der LDH-Aktivität ausmachen und die Ausbeute an LDH-4 und LDH-5 für diese Untersuchungen zu gering war. Die Michaeliskonstanten wurden nach LINEwEAver und Burk' (15) bestimmt und ergaben bei einer Reaktionstemperatur von $37^{\circ} \mathrm{C}$ deutlich unterschiedliche Werte (siehe Legende $z u$ Abb. 4). In den pS-Aktivitäts-Diagrammen (Abb. 4) treten die Unterschiede der Substratsättigungsfunktionen der LDH-Isoenzyme noch deutlicher hervor. Die optimalen Substratkonzentrationen der LDH-Isoenzyme für Pyruvat und Lactat nehmen von LDH-1 nach LDH-3 zu. Bei der Hemmung der Isoenzyme durch Lactatüberschuß zeigt sich ein von LDH-1 nach LDH3 ansteigender Hemmungstyp höherer Ordnung. Die pS-Aktivitäts-Diagramme der Isoenzyme bei der Pyruvatreduktion entsprechen angenähert symmetrischen Glockenkurven. Für diese Kurvenform ist nach NETTER (16) ein kompetitiver Hemmungstyp anzunehmen.
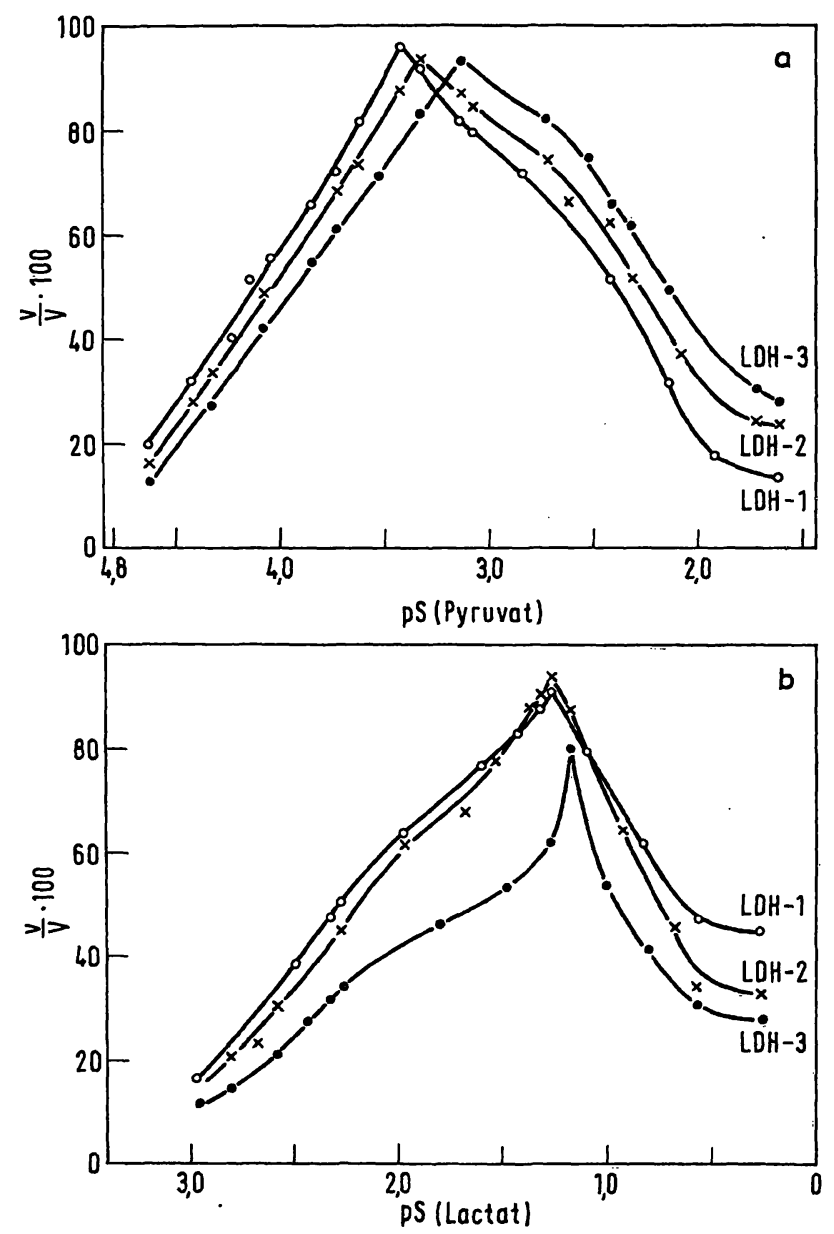

Abb. 4

Aktivitäts-pS-Diagramme der Isoenzyme LDH-1, LDH-2 und LDH-3 des Myokards des Kalbsherzens, Substrate: a) Pyruvat und b) Lactat. Michaeliskonstanten der Isoenzyme für Lactat: LDH-1 $=5,27$, $\mathrm{LDH}-2=0,092, \mathrm{LDH}-3=0,122 \mathrm{mmol} / \mathrm{l}$. Substratoptima der Isoenzyme für Lactat: LDH-1 $=53,3, \mathrm{LDH}-2=53,3, \mathrm{LDH}-3=66,7$ und für Pyruvat LDH-1 $=0,38, \mathrm{LDH}-2=0,48$ und $\mathrm{LDH}-3=0,72 \mathrm{mmol} / \mathrm{l}$. Hemmkonstanten der Isoenzyme bei Pyruvatüberschuß: LDH-1 $=4,0$ $\mathrm{LDH}-2=4,5, \mathrm{LDH}-3=5,4 \mathrm{mmol} / 1$ (13)
Zusätzlich bestimmten wir auch die Hemmkonstanten der Isoenzyme für Pyruvat. Die Auswertung erfolgte nach Drxon (17). Wir fanden unterschiedliche, von LDH-1 nach LDH-3 ansteigende Hemmkonstanten (siehe Legende zu Abb. 4). Diese Hemmung beruht auf der Bildung eines ternären Komplexes aus LDHSubunit, Pyruvat und NAD $(18,19)$ wobei das Pyruvat wahrscheinlich in seiner Enolform reagiert (20).

\section{Diskussion}

Im Myokard und Reizleitungssystem des Kalbs- und Rinderherzens konnten mit der Disc-Elektrophorese in Polyacrylamid-Gel und der gängigen Tetrazoliumfärbemethode, bei der Lactat als Substrat verwendet wird, drei LDH-Isoenzyme nachgewiesen werden. Beide Organanteile besitzen ein sog. oxidatives Isoenzymmuster, das durch das Überwiegen der anodischen LDH-Isoenzyme charakterisiert ist. Im Reizleitungssystem macht LDH-1 den größten Anteil an der LDH-Gesamtaktivität aus, im Myokard LDH-2.

Nach chromatographischer Trennung der LDH-Isoenzyme des Myokards und dem Nachweis mit Pyruvat als Substrat fanden wir ein Isoenzymmuster, das durch das Úberwiegen der LDH-1 gekennzeichnet ist. Dieser Befund stimmt mit den Ergebnissen der Untersuchungen anderer Autoren überein, die die LDH-Isoenzyme nach chromatographischer oder elektrophoretischer Trennung mit Pyruvat als Substrat nachwiesen (3, 4, 8, 12, 22).

Während wir unter den Standardbedingungen der Polyacrylamid-Disc-Elektrophorese in beiden Organen nur drei Isoenzyme nachweisen konnten, stellten sich bei Verwendung hoher Lactatkonzentrationen im Färbemedium auch die Isoenzyme LDH-4 und LDH-5 dar, ein Befund, der durch die unterschiedlichen kinetischen Eigenschaften der LDH-Isoenzyme zu erklären ist $(3,9,10,23)$. In den Extrakten des Arbeitsmyokards und Reizleitungssystems lassen sich mit niedrigen Lactatkonzentrationen, die im Bereich der Substratoptima der anodischen Isoenzyme liegen, die kathodischen Isoenzyme nicht darstellen. Mit steigender Lactatkonzentration werden die kathodischen Isoenzyme stetig aktiver (vgl. Abb. 2), während die anodischen Isoenzyme zunehmend gehemmt werden. Bei Aufnahme der Substratsättigungsfunktion der Isoenzyme für Lactat fanden wir von LDH-1 nach LDH-3 ansteigende Substratoptima, aber eine von LDH-1 nach LDH-3 zunehmende Hemmung durch höhere Lactatkonzentrationen (Abb. 4). Im optischen Test wurde im Gegensatz zur spezifischen Anfärbung mit der Tetrazoliummethode in der Testlösung kein Cyanid eingesetzt, das entstandenes Pyruvat unter Hydroxynitrilbildung umsetzen und damit eine Hemmung der LDH-Isoenzyme durch ternäre Komplexbildung aus Pyruvat, NAD und Subunit verhindern kann.

Verschiedene Methoden der analytischen und präparativen Darstellung von LDH-Isoenzymen können 
ebenfalls zu unterschiedlichen Ergebnissen führen. Die vor der chromatographischen Trennung durchgeführte Vorfraktionierung mit Ammoniumsulfat sowie das Einengen und Dialysieren der Probe führen zu einer Inaktivierung von M-Polypeptiden der Lactatdehydrogenase. Dieser Vorgang ist möglicherweise mit Dissoziation und Rekombination von Untereinheiten (24) verbunden und kann so einen Abfall der Isoenzymaktivitäten, der von LDH-2 nach LDH-5 zunimmt, bedingen $(25,26)$. Die Dauer der chromatographischen Trennung (4-5 Tage) und die Verdünnung der Probe im Verlauf der Trennung bedingen ebenfalls eine Inaktivierung von M-Polypeptiden, die gegenüber Verdünnung, Lagerung, Temperaturerhöhung und Erniedrigung besonders empfindlich sind $(23,26,27,28)$. Die Bestimmung des LDH-Isoenzymmusters mit der Polyacrylamid-Disc-Elektrophorese nimmt dagegen wesentlich geringere Zeit in Anspruch und es fehlen die Konzentrierungs- und Verdünnungsschritte bei der Präparation und Trennung des Extraktes, so daß eine ins Gewicht fallende Inaktivierung von M-Polypeptiden weitgehend ausgeschlossen werden kann.

Außer den genannten Faktoren, die das gewonnene $\mathrm{LDH}-$ Isoenzymmuster beeinflussen, fanden andere Autoren $(27,29,30)$, daß die Extraktionsart, eingesetzte Enzymaktivität, Elektrophoresebedingungen und Dauer der Färbung ebenfalls einen Einfluß auf das gewonnene LDH-Isoenzymmuster haben. Das mit einer Methode erhaltene LDH-Isoenzymmuster eines Organs hängt damit wesentlich von den gewählten methodischen Be- dingungen $a b$. Isoenzymmuster, die mit verschiedenen Methoden gewonnen wurden, sind daher nicht ohne weiteres vergleichbar.

Zusätzliche Informationen erhielten wir aus der kinetischen Analyse. Aus den pS-Aktivitäts-Diagrammen (Abb. 2) geht hervor, daß der Anteil der Isoenzyme $\mathrm{LDH}-4$ und LDH-5 an der LDH-Gesamtaktivität im Reizleitungssystem geringer ist als im Myokard. Beide Organe weisen ein sog. oxidatives Isoenzymmuster auf, wie es für Organe mit vorwiegend oxidativem Stoffwechsel kennzeichnend sein soll (8). Nach histochemischen Untersuchungen (31) und der Bestimmung des Enzymverteilungsmusters im Reizleitungssystem und Myokard (32) ist anzunehmen, daß das Reizleitungssystem einen geringeren Energiebedarf und eine geringere Energiebereitstellung hat als das Myokard. Kübler et al. (32) deuten ihre Befunde so, daß im Reizleitungssystem das Verhältnis von oxidativer $z u$ anaerober Energiebereitstellung im Vergleich zum Arbeitsmyokard zugunsten der Glykolyse verschoben ist. Unter der Voraussetzung niedrigerer glykolytischer Flußraten in den Purkinjezellen - im Vergleich zum Skeletmuskel - können die anodischen Isoenzyme LDH-1, LDH-2 und LDH-3 im Hinblick auf ihre niedrigen Michaeliskonstanten und Substratoptima gerade bei niedrigen Pyruvatkonzentrationen ausreichend Pyruvat in Lactat umsetzen. Deshalb dürfte das LDHIsoenzymmuster des Reizleitungsssystems nicht nur von seinem Stoffwechsel typ, sondern vor allem durch seine Stoffwechselaktivität (33) bestimmt sein.

\section{Literatur}

1. Vesell, E. S. \& Bearn, A. G (1957), Proc. Soc. Exp. Biol. Med. 94, 96-99. - 2. WIEME, R. J. (1959), Clin. Chim. Acta 4, 46-50. - 3. Veseld, E. S. \& Bearn, A. G. (1961), J. Clin. Invest. 40, 586-591. - 4. Wròblewski, F. (1963), Progress in Cardiovascular Diseases 6, 63-83. - 5. LehmanN, F. G., Schneider, K. W., Schering, G. \& Koch, K. (1966), Verh. Deut. Ges. Inn. Med. 72, 709-712. - 6. Pearson, C. M., Kar, N. C., Peter, J. P. \& Munsat, T. L. (1965), Ä̈ner. J. Med. 39, 91-97. - 7. Langvad, E. (1968), Eur. J. Cancer 4, 107-115. 8. Pfleiderer, G. \& Wachsmuth, E. D. (1961), Biochem. Z. 334, 185-197. - 9. Cahn, R. D., Kaplan, N. O., Levine, L. \& Zwilling, E. (1962), Science 136, 962-969. - 10. Dawson, D. M., Goodfriend, T. L. \& Kaplan, N. O. (1964), Science 143, 929-933. - 11. Vesell, E. S. (1965), Science 150, 1735 bis 1737. - 12. Dietz, A. A. \& Lubrano, Tina (1967), Anal. Biochem. 20, 246-257. - 13. DöLKEN, G. (1971), Inaugural-Diss. Köln. 14. Schumacher, K., Schneider, W. \& Gross, R. (1968), Thrombose Diath. Haemorrh. 20, 430-437. - 15. LinewEAVER, H. \& Burk, D. (1934), J. Amer. Chem. Soc. 56, 658-666. 16. Netrer, H. (1959), Theoretische Biochemie, Springer Verlag, Berlin-Heidelberg-Göttingen, S. 583 ff. - 17. DrxoN, M. (1953), Biochem. J. 55, 170-171. - 18. Gutrreund, H., Cantwell, R., McMurray, H. M., Criddle, R. S. \& Hathaway, G. (1968),
Biochem. J. 106, 683-687. - 19. TARMY, E. M. \& Kaplan, N. O. (1968), J. Biol. Chem. 243, 2587-2596. - 20. Griffin, J. H. \& R. S. Criddle (1970), Biochemistry 9, 1195-1205. - 21. PlageMANN, P. G. W., GREGORY, K. F. \& WRÒBLEWSKI, F. (1960), J. Biol. Chem. 235, 2282-2287. - 22. Hess, B. \& WALTER, S. J. (1960), Klin. Wochenschr. 38, 1080-1088. - 23. Plagemann, P. G. W., GREGoRY, K. F. \& WRòblewsKr, F. (1960), J. Biol. Chem. 235, 2288-2293. - 24. Millar, D. B., SUmmers, M. R. \& Niziolek, J. A. (1971), Nature New Biol. 230, 117-119. 25. Wachsmuth, E. D. \& Pfleiderer, G. (1963), Biochem. Z. 336, 545-556. - 26. Hrur, R. B. \& MEACHAN, E. J. (1961), Ann. N. Y. Acad. Sci. 94, 868-876. - 27. Vesele, E. S. (1962), Nature (London) 195, 497-498. - 28. ZondAG, H. A. (1963), Science 142, 965-967. - 29. Ressler, N., Schulz, J. L. \& JoSEPH, R. (1963), Nature (London) 198, 888-889. - 30. VESELL, E. S. \& Brody, I. A. (1964), Ann. N. Y. Acad. Sci. 121, 544-560. 31. Schiebler, T. H., Stark, M. \& Caesar, R. (1956), Klin. Wochenschr. 34, 181-183. - 32. KüBLER, W., von SMEKAL, P., Gerhard, W. \& Schumacher, K. (1969), Verh. Dtsch. Ges. Kreisl.-Forsch. 35, 169-174. - 33. DölkeN, G., Schumacher, K., KübleR, W., SChNeider, W. \& Gross, R. (1971), Verh. Dtsch. Ges. Inn. Med. 77, 164-166.

\author{
Dr. med. Gottfried Dölken \\ z. Z. Fachbereich Biologie der Universität Konstanz \\ 775 Konstanz \\ Prof. Dr. K. Schumacher \\ 5 Köln 41 \\ Medizinische Universitätsklinik Köln-Lindenthal \\ Josef-Stelzmann-Str. 9
}

